\title{
Residual A Posteriori Error Estimator for a Three-Field Model of a Non-linear Generalized Stokes Problem
}

\author{
Vincent J. Ervin* Timothy N. Phillips ${ }^{\dagger}$
}

\begin{abstract}
In this article we propose and analyze an a posteriori error estimator for a three-field model of a generalized Stokes problem. The components of the a posteriori error estimator are defined via a non-linear projection of the residues of the variational equations. Both upper and lower bounds for the approximation error are derived in terms of the components of the a posteriori error estimator. The non-linear projections do not need to be explicitly computed to construct the a posteriori error estimates.
\end{abstract}

Key words. a posteriori error estimation, power law fluid, generalized Stokes problem

AMS Mathematics subject classifications. 65N30

\section{Introduction}

A posteriori error estimates are an important tool in solving fluid flow problems, whose solutions can exhibit complicated structures. An a posteriori error estimate can be used as an indicator for guiding adaptive numerical methods. Computational effort can then be efficiently channelled to those regions in the flow domain that require particular resolution of fine-scale structures. For most physical problems, simple uniform refinement across the entire computational domain can quickly exhaust computational resources, often without determining a satisfactory approximation.

The three-field model of a generalized Stokes problem may be considered as a prototype for viscoelastic problems since the mathematical description of a viscoelastic fluid is invariably in terms of three fields due to the nonlinear constitutive equation relating the stress to the strain. Very little work has been done on a posteriori error estimation for viscoelastic flow problems. Here, the need for affordable and reliable error indicators is even greater than for Newtonian flow problems due to the larger number of dependent variables required in the mathematical formulation. For two-dimensional planar flows there are six dependent variables for viscoelastic problems compared with three for Newtonian problems when within the primitive variable formulation.

The estimation procedures that have been proposed to date for viscoelastic flows can be divided into two classes: element residual methods (see [22], for example) and subdomain residual methods

\footnotetext{
*Department of Mathematical Sciences, Clemson University, Clemson, SC, 29634-0975, USA. email: vjervin@clemson.edu. Partially supported by the NSF under grant no. DMS-0410792. This research was undertaken while a visitor in the Institute of Mathematical and Physical Sciences, University of Wales, Aberystwyth.

${ }^{\dagger}$ School of Mathematics, Cardiff University, Senghennydd Road, Cardiff CF24 4AG, United Kingdom. email: PhillipsTN@cf.ac.uk
} 
[12]. Evidence for the appropriateness of these methods as a means of obtaining reliable error estimators has been empirical to date and there is, therefore, an urgent need to establish reliable error estimators for this class of fluid flow problems. The majority of papers dealing with error estimates for viscoelastic flow problems include estimates of the stress errors in their error estimates. An exception to this is the work of Mutlu et al. [17], who used an a posteriori error estimate based solely on the velocity gradients to guide the adaptive strategy.

Yurun and Crochet [26] have shown that, even in the absence of theoretical justification, the use of element residual methods for error estimation in adaptive procedures can lead to solutions of highly elastic liquids through complex geometries at costs which are less than those incurred with uniform meshes. In this approach a $p$-adaptive high-order streamline-upwinded Petrov-Galerkin finite element method was used for the simulation of the flow of a UCM fluid through a $4: 1$ axisymmetric smooth contraction. The maximum residual error at each of the nodes in a given element was taken as the elemental error indicator and solutions beyond a Weissenberg number $\mathrm{We}=10$ were obtained without difficulty. However, with the adaptive procedure which they used the rate at which the residual error was reduced decreased with polynomial enrichment leading the authors to conclude that a better adaptive procedure based on reliable error estimates was needed.

An element residual method for viscoelastic flows proposed by Warichet and Legat [25] was an obvious example of an attempt to extend the element residual method used by Ainsworth and Oden [1] for the Stokes problem. No theoretical results are given by the authors and evidence for the success of their estimators, as with others in the viscoelastic literature, is purely empirical. These ideas were further developed by Owens [19] and Chauvière and Owens [5] in an attempt to provide some theoretical undergirding to the choice of appropriate error indicators for viscoelastic problems. In the latter paper it is shown that the local elemental error residual for the elastic stresses only accounts for the so-called stress cell error and therefore is not generally an adequate measure of the local error. An improved error indicator, which takes into account the transmitted error, is proposed and computed on an element-by-element basis.

In [7] Gatica et al. proposed and analysed a posteriori error estimators for a low-order method for generalized Stokes flow. The formulation used as a four-field approach in which the gradient of the velocity was treated as an additional unknown. They applied a Bank-Weiser type analysis to construct reliable and quasi-efficient a posteriori error estimates for their mixed finite element approximations.

In this paper we propose a residual based a posteriori error estimate for a three-field model of a generalized Stokes problem. Largely motivating our investigation is the numerical approximation of fluids having a power law constitutive equation, i.e.

$$
\sigma=\nu_{0}|d(\mathbf{u})|^{r-2} d(\mathbf{u}), \quad \nu_{0}>0, \quad 1<r<2,
$$

where $\sigma$ denotes the extra stress tensor, $\mathbf{u}$ the fluid velocity, and $d(\mathbf{u}):=\left(\nabla \mathbf{u}+\nabla \mathbf{u}^{T}\right) / 2$ the rate of deformation tensor. (Here and throughout the paper we use the following notation: $\nabla \mathbf{u}$ is the tensor gradient of $\mathbf{u}$, for tensors $\sigma=\left(\sigma_{i j}\right), \tau=\left(\tau_{i j}\right), \sigma: \tau=\sum_{i, j} \sigma_{i j} \tau_{i j},|\sigma|^{2}=\sigma: \sigma$. Also, for $r>1$ we denote its unitary conjugate by $r^{\prime}$, satisfying $r^{-1}+r^{\prime-1}=1$.)

The power law model has been used to model the viscosity of many polymeric solutions and melts over a considerable range of shear rates [11].

Other constitutive equations having a similar form to the power law model include $[4,15,20]$ : 
Ladyzhenskaya Law[14]:

$$
\sigma=\nu_{0}+\nu_{1}|\nabla \mathbf{u}|^{r-2} d(\mathbf{u}), \quad \nu_{0} \geq 0, \nu_{1}>0, r>1
$$

used in modeling fluids with large stresses.

Carreau Law:

$$
\sigma=\nu_{0}\left(1+|d(\mathbf{u})|^{2}\right)^{(r-2) / 2} d(\mathbf{u}), \quad \nu_{0}>0, r \geq 1,
$$

used in modeling visco-plastic flows and creeping flow of metals.

The a posteriori error estimators we propose in this paper are extensions of those studied in [1] (for the Stokes and Oseen equations) and similar to those given in Sandri [23], with the difference being that the residual functions lie in different spaces. In [23] the residual functions were defined in the dual spaces of the test functions. Herein, via non-linear projections, we introduce residual functions defined in the same spaces as the test (and trial) functions. However, one does not need to compute the non-linear projections in order to construct the a posteriori error estimates.

The error estimates we derive involve the residual of the variational equations (variational residues), as opposed to the residual of the modeling equations (strong residues). The variational residues differ from the strong residues in that powers of the mesh parameter $h$ do not appear as multipliers of various terms in the expression. The constant multipliers in the variational residual estimates only depend upon the coercivity and continuity constants ( $c$ and $M$, in (2.9),(2.10)) and the two inf-sup constants $\left(\gamma_{V P}\right.$ and $\gamma_{V S}$, in $\left.(2.14),(2.15)\right)$. The constants appearing in the strong residual estimates additionally involve the norm of the inverse of the linearized adjoint problem and interpolation constants. We also note that our error estimates are established independently of how the numerical approximation $\left(\sigma^{N}, \mathbf{u}^{N}, p^{N}\right)$ of the exact solution $(\sigma, \mathbf{u}, p)$ is obtained. In, other words, the residual functionals need not satisfy any sort of Galerkin orthogonality property.

Though our investigations were largely motivated by the power law model, our analysis presented below is for a general constitutive model satisfying assumptions A1, A2, and A3, described below.

The paper is organized as follows. In the next section we introduce the generalized Stokes problem, notation, and state our assumptions regarding the modeling equations. The error estimators are introduced in Section 3 and their relationship to the true error investigated. In Section 4 we discuss issues related to the actual computation of the a posteriori error estimators.

\section{Mathematical Model}

Mathematical Notation

Used in the analysis below are the following function spaces and norms.

$$
T:=\left(L^{r^{\prime}}(\Omega)\right)_{s}^{d \times d}=\left\{\tau=\left(\tau_{i j}\right) ; \tau_{i j}=\tau_{j i} ; \tau_{i j} \in L^{r^{\prime}}(\Omega) ; i, j=1, \ldots, \dot{d}\right\}
$$

with norm $\|\tau\|_{T}:=\left(\int_{\Omega}|\tau|^{r^{\prime}} d \Omega\right)^{1 / r^{\prime}}$. (The subscript $s$ is used to denote the subspace of symmetric tensors.) 
$V:=\left(W_{0}^{1, r}(\Omega)\right)^{d}$, with $W^{k, p}(\Omega)$ denoting the usual Sobolev space notation. We take for the norm on $V,\|v\|_{V}:=\left(\int_{\Omega}|\nabla v|^{r} d \Omega\right)^{1 / r}$, which is equivalent to the usual $\|\cdot\|_{W^{1, r}}$ norm by the PoincaréFriedrichs lemma.

$$
P:=L_{0}^{r^{\prime}}(\Omega)=\left\{q \in L^{r^{\prime}}(\Omega): \int_{\Omega} q d \Omega=0\right\},
$$

with norm $\|q\|_{P}:=\left(\int_{\Omega}|q|^{r^{\prime}} d \Omega\right)^{1 / r^{\prime}}$.

For a Banach space $X, X^{\prime}$ denotes its dual space with associated norm $\|\cdot\|_{X^{\prime}}$. For $\sigma, \tau$ tensors, $\mathbf{u}$, $\mathbf{v}$ vectors, $f, g$, scalars, we use $\langle\cdot, \cdot\rangle$ to denote the scalar quantities $\langle\sigma, \tau\rangle:=\sigma: \tau,\langle\mathbf{u}, \mathbf{v}\rangle:=\mathbf{u} \cdot \mathbf{v}$, and $\langle f, g\rangle:=f g$.

\section{Model Problem}

Let $\Omega$ denote a bounded domain in $\mathbb{R}^{d},(\dot{d}=2,3)$, with boundary $\partial \Omega$. We take as our model problem: Given the body force term $\mathbf{f}$, determine $\sigma, \mathbf{u}, p$ satisfying

$$
\begin{aligned}
& \sigma=g(\mathbf{u}) \quad \text { in } \Omega, \\
& -\nabla \cdot \sigma+\nabla p=\mathbf{f} \quad \text { in } \Omega, \\
& \nabla \cdot \mathbf{u}=0 \text { in } \Omega \text {, } \\
& \mathbf{u}=0 \quad \text { on } \partial \Omega \text {. }
\end{aligned}
$$

In (2.1)-(2.4) $\sigma$ represents the extra stress tensor, $\mathbf{u}$ the fluid velocity, and $p$ the fluid pressure. Equation (2.1) represents a constitutive model for the fluid (see (1.1)-(1.3) for examples).

We make the following assumption for the constitutive modeling equation (2.1).

A1: $g(\mathbf{u})$ is (formally) uniquely invertible to obtain

$$
d(\mathbf{u})=\breve{g}(\sigma) \sigma \quad, \quad(\text { or } \nabla \mathbf{u}=\check{g}(\sigma) \sigma)
$$

and the inverse is continuous.

For the power law fluid (1.1), taking the inner-product of both sides of the equation with itself we obtain

$$
|\sigma|^{2}=\nu_{0}^{2}|d(\mathbf{u})|^{2 r-2} .
$$

Combining (2.5) with (1.1) we obtain

$$
d(\mathbf{u})=\nu_{0}^{1-r^{\prime}}|\sigma|^{r^{\prime}-2} \sigma
$$

as described by $\mathbf{A} \mathbf{1}$.

Introduce the functionals $a: T \times T \rightarrow \mathbb{R}, b: T \times V \rightarrow \mathbb{R}, s: V \times P \rightarrow \mathbb{R}$, defined by

$$
\begin{aligned}
a(\sigma, \tau) & :=\int_{\Omega} \breve{g}(\sigma) \sigma: \tau d \Omega, \\
b(\tau, \mathbf{u}) & :=\int_{\Omega} \tau: d(\mathbf{u}) d \Omega \\
s(\mathbf{v}, p) & :=\int_{\Omega} \nabla \cdot \mathbf{v} p d \Omega .
\end{aligned}
$$


Additionally, we assume the following coercivity and continuity properties for $G(\sigma):=\breve{g}(\sigma) \sigma$.

$$
\text { A2: } \begin{aligned}
\left(G\left(t_{1}\right)-G\left(t_{2}\right)\right):\left(t_{1}-t_{2}\right) & \geq c\left|t_{1}-t_{2}\right|^{r^{\prime}}, \quad \forall t_{1}, t_{2} \in \mathbb{R}^{\dot{d} \times \dot{d}} \\
\text { A3: }\left|G\left(t_{1}\right)-G\left(t_{2}\right)\right| & \leq M\left(\left|t_{1}\right|+\left|t_{2}\right|\right)^{r^{\prime}-2}\left|t_{1}-t_{2}\right|, \quad \forall t_{1}, t_{2} \in \mathbb{R}^{\dot{d} \times \dot{d}} .
\end{aligned}
$$

For the power law fluid properties A2, A3, follow from ([6],pg. 319)

$$
\begin{aligned}
\left(\|\xi\|^{p-2} \xi-\|\eta\|^{p-2} \eta\right) \cdot(\xi-\eta) & \geq C\|\xi-\eta\|^{p}, \quad \xi, \eta \in \mathbb{R}^{\dot{d}}, p \geq 2, c>0, \\
\|\| \xi\left\|^{p-2} \xi-\right\| \eta\left\|^{p-2} \eta\right\| & \leq M(\|\xi\|+\|\eta\|)^{p-2}\|\xi-\eta\|, \quad \forall \xi, \eta \in \mathbb{R}^{\dot{d}}, p \geq 2 .
\end{aligned}
$$

We will assume that the body force term $\mathbf{f}$ in the momentum equation represents a conservative vector field. Then, there exists a scalar function $f_{p}$ (unique up to a constant) such that

$$
\mathbf{f}=\nabla f_{p}
$$

In Section 4 we discuss the modification required when $\mathbf{f}$ is not a conservative vector field.

Using the above notation, the variational formulation of (2.1)-(2.4) may be stated as: Given a conservative vector field $\mathbf{f} \in\left(W_{0}^{-1, r^{\prime}}(\Omega)\right)^{d}$, determine $(\sigma, \mathbf{u}, p) \in T \times V \times P$ such that

$$
\begin{aligned}
a(\sigma, \tau)-b(\tau, \mathbf{u}) & =0, \quad \forall \tau \in T \\
b(\sigma, \mathbf{v})-s(\mathbf{v}, p) & =\int_{\Omega} f_{p} \nabla \cdot \mathbf{v} d \Omega, \quad \forall \mathbf{v} \in V \\
s(\mathbf{u}, q) & =0, \quad \forall q \in P .
\end{aligned}
$$

Associated with the unique solvability of (2.11)-(2.13) are the two inf-sup conditions:

Velocity-Pressure inf-sup:

There exists $\gamma_{V P}>0$ such that

$$
\inf _{q \in P} \sup _{\mathbf{v} \in V} \frac{s(\mathbf{v}, p)}{\|\mathbf{v}\|_{V}\|q\|_{P}} \geq \gamma_{V P}
$$

Velocity-Stress inf-sup:

There exists $\gamma_{V S}>0$ such that

$$
\inf _{\mathbf{v} \in V} \sup _{\tau \in T} \frac{b(\tau, \mathbf{v})}{\|\tau\|_{T}\|\mathbf{v}\|_{V}} \geq \gamma_{V S}
$$

For $T^{N} \subset T, V^{N} \subset V, P^{N} \subset P$, the usual three-field numerical approximation to (2.11)-(2.13) is given by (see [7, 15] for alternate approximation methods): Determine $\left(\sigma^{N}, \mathbf{u}^{N}, p^{N}\right) \in T^{N} \times V^{N} \times P^{N}$ such that

$$
\begin{aligned}
a\left(\sigma^{N}, \tau\right)-b\left(\tau, \mathbf{u}^{N}\right) & =0, \quad \forall \tau \in T^{N}, \\
b\left(\sigma^{N}, \mathbf{v}\right)-s\left(\mathbf{v}, p^{N}\right) & =\int_{\Omega} f_{p} \nabla \cdot \mathbf{v} d \Omega, \quad \forall \mathbf{v} \in V^{N}, \\
s\left(\mathbf{u}^{N}, q\right) & =0, \quad \forall q \in P^{N} .
\end{aligned}
$$


Analogous to (2.14) and (2.15), associated with (2.16)-(2.16) are the following inf-sup conditions on the approximation spaces $T^{N}, V^{N}$, and $P^{N}$.

Discrete Velocity-Pressure inf-sup:

There exists $\tilde{\gamma}_{V P}>0$ such that

$$
\inf _{q \in P^{N}} \sup _{\mathbf{v} \in V^{N}} \frac{s(\mathbf{v}, p)}{\|v\|_{V}\|q\|_{P}} \geq \tilde{\gamma}_{V P}
$$

Discrete Velocity-Stress inf-sup:

There exists $\tilde{\gamma}_{V S}>0$ such that

$$
\inf _{\mathbf{v} \in V^{N}} \sup _{\tau \in T^{N}} \frac{b(\tau, \mathbf{v})}{\|\tau\|_{T}\|v\|_{V}} \geq \tilde{\gamma}_{V S}
$$

Existence and uniqueness of solutions to (2.11)-(2.13), and (2.16)-(2.18) were studied in [2], and a priori error estimates for the error in the approximations derived for $d=2$. Boundedness of the approximation $\left(\sigma^{N}, \mathbf{u}^{N}, p^{N}\right)$ follow automatically from the boundedness of $(\sigma, \mathbf{u}, p)$ and the a priori error estimate. Central to the analysis of (2.11)-(2.13), and (2.16)-(2.18) are the inf-sup conditions. The discrete velocity-pressure inf-sup condition (2.19) also occurs in the approximation of Stokes and Navier-Stokes problems. As such, spaces $V^{N}$ and $P^{N}$ satisfying (2.19) are well studied (see for example $[9,10])$. For the traditional $h$-approximation method spaces satisfying $(2.20)$ have been investigated in $[23,15]$. For the spectral approximation method compatible spaces have been investigated in [8].

\section{A Posteriori Error Estimators}

In this section we introduce the a posteriori error estimators and show that they form both upper and lower bounds to the error in the approximations, in suitable norms.

Define the functionals $\mathbb{A}: T \times T \rightarrow \mathbb{R}, \mathbb{B}: V \times V \rightarrow \mathbb{R}, \mathbb{S}: P \times P \rightarrow \mathbb{R}$, as:

$$
\begin{aligned}
\mathbb{A}(\Upsilon, \tau) & :=\int_{\Omega}|\Upsilon|^{r^{\prime}-2} \Upsilon: \tau d \Omega ; \\
\mathbb{B}(\mathbf{R}, \mathbf{v}) & :=\int_{\Omega}|\nabla \mathbf{R}|^{r-2} \nabla \mathbf{R}: \nabla \mathbf{v} d \Omega \quad ; \quad \mathbf{R} \in V, \mathbf{v} \in V, \\
\mathbb{S}(R, q) & :=\int_{\Omega}|R|^{r^{\prime}-2} R q d \Omega \quad ; \quad R \in P, q \in P .
\end{aligned}
$$

Note that by Hölder's inequality

$$
\begin{aligned}
\int_{\Omega}|\Upsilon|^{r^{\prime}-2} \Upsilon: \tau d \Omega & \leq\left(\int_{\Omega}\left(|\Upsilon|^{r^{\prime}-2}|\Upsilon|\right)^{r} d \Omega\right)^{1 / r}\left(\int_{\Omega}|\tau|^{r^{\prime}} d \Omega\right)^{1 / r^{\prime}} \\
& =\left(\int_{\Omega}|\Upsilon|^{\left(r^{\prime}-1\right) r} d \Omega\right)^{1 / r}\|\tau\|_{T} \\
& =\|\Upsilon\|_{T}^{r^{\prime} / r}\|\tau\|_{T} .
\end{aligned}
$$


Thus, $\mathbb{A}: T \times T \rightarrow \mathbb{R}$ is well defined. Similarly, we have that $\mathbb{B}$ and $\mathbb{S}$ are also well defined.

For the functionals $\mathbb{A}, \mathbb{B}$ and $\mathbb{S}$ the choice of the kernels (i.e. $|\Upsilon|^{r^{\prime}-2},|\nabla \mathbf{R}|^{r-2}$, and $|R|^{r^{\prime}-2}$, respectively) are chosen such that both arguments lie in the same (appropriate) functional space.

Next, observe that

$$
\mathbb{A}(\Upsilon, \Upsilon)=\|\Upsilon\|_{T}^{r^{\prime}}, \quad \mathbb{B}(\mathbf{R}, \mathbf{R})=\|\mathbf{R}\|_{V}^{r}, \quad \mathbb{S}(R, R)=\|R\|_{P}^{r^{\prime}}
$$

Let $\epsilon:=\sigma-\sigma^{N}, \mathbf{e}:=\mathbf{u}-\mathbf{u}^{N}$, and $e:=p-p^{N}$.

We now introduce residual functions $(\Upsilon, \mathbf{R}, R) \in T \times V \times P$, and corresponding residual functionals $\left(\mathcal{R}_{1}, \mathcal{R}_{2}, \mathcal{R}_{3}\right): T \times V \times P \rightarrow \mathbb{R}$, defined by

$$
\begin{aligned}
\mathbb{A}(\Upsilon, \tau):=a(\sigma, \tau)-a\left(\sigma^{N}, \tau\right)-b(\tau, \mathbf{e}) & =\mathcal{R}_{1}(\tau):=-a\left(\sigma^{N}, \tau\right)+b\left(\tau, \mathbf{u}^{N}\right), \\
\mathbb{B}(\mathbf{R}, \mathbf{v}):=b(\epsilon, \mathbf{v})-s(\mathbf{v}, \mathbf{e}) & =\mathcal{R}_{2}(\mathbf{v}):=\int_{\Omega} f_{p} \nabla \cdot \mathbf{v} d \Omega-b\left(\sigma^{N}, \mathbf{v}\right)+s\left(\mathbf{v}, p^{N}\right) \\
\mathbb{S}(R, q):=s(\mathbf{e}, q) & =\mathcal{R}_{3}(q):=-s\left(\mathbf{u}^{N}, q\right)
\end{aligned}
$$

for all $(\tau, \mathbf{v}, q) \in T \times V \times P$. The existence of $(\Upsilon, \mathbf{R}, R) \in T \times V \times P$ follows from the theory of monotone operators (see [27], also [6]).

Define an "error magnitude", $\|||(\epsilon, \mathbf{e}, e)\|_{*}^{*}$, as

$$
\|\mid(\epsilon, \mathbf{e}, e)\|_{*}^{*}=\mathbb{A}(\Upsilon, \Upsilon)+\mathbb{B}(\mathbf{R}, \mathbf{R})+\mathbb{S}(R, R) .
$$

In passing, we make the following observation. For the functional $J: T \times V \times P \rightarrow \mathbb{R}$ defined by

$$
\begin{aligned}
J(\tau, \mathbf{v}, q):= & \frac{1}{2}\left(\frac{1}{r^{\prime}} \mathbb{A}(\tau, \tau)+\frac{1}{r} \mathbb{B}(\mathbf{v}, \mathbf{v})+\frac{1}{r^{\prime}} \mathbb{S}(q, q)\right) \\
& -\left(a(\sigma, \tau)-a\left(\sigma^{N}, \tau\right)-b(\tau, \mathbf{e})+b(\epsilon, \mathbf{v})-s(\mathbf{v}, \mathbf{e})+s(\mathbf{e}, q)\right),
\end{aligned}
$$

it is straightforward to show that

$$
-\frac{1}{2}\|(\epsilon, \mathbf{e}, e)\|_{*}^{*}=J(\Upsilon, \mathbf{R}, R)=\inf _{(\tau, \mathbf{v}, q) \in T \times V \times P} J(\tau, \mathbf{v}, q) .
$$

\subsection{Relationship between $(\epsilon, \mathbf{e}, e)$ and $(\Upsilon, \mathbf{R}, R)$}

In this section we show the relationship between the residual functions and the true error. These relationships are summarized in the following theorem.

Theorem 3.1 For $(\sigma, \mathbf{u}, p)$ satisfying (2.11)-(2.13) and $\left(\sigma^{N}, \mathbf{u}^{N}, p^{N}\right) \in T \times V \times P$ an approximation, with $(\Upsilon, \mathbf{R}, R)$ defined in (3.5)-(3.7) we have that there exists a constant $C$ independent of the approximation parameters such that

$$
\begin{aligned}
\left\|\sigma-\sigma^{N}\right\|_{T}^{r^{\prime}} & \leq C\left(\|\Upsilon\|_{T}^{r^{\prime}}+\|\mathbf{R}\|_{V}^{r^{2} / r^{\prime}}+\|\mathbf{R}\|_{V}^{r}+\|R\|_{P}^{r^{\prime}}\right), \\
\left\|\mathbf{u}-\mathbf{u}^{N}\right\|_{V}^{r} & \leq C\left(\|\Upsilon\|_{T}^{r^{\prime}}+\|\Upsilon\|_{T}^{r}+\|\mathbf{R}\|_{V}^{r^{3} / r^{\prime 2}}+\|\mathbf{R}\|_{V}^{r^{2} / r^{\prime}}+\|R\|_{P}^{r}\right), \\
\left\|p-p^{N}\right\|_{P}^{r^{\prime}} & \leq C\left(\|\Upsilon\|_{T}^{r^{\prime}}+\|\mathbf{R}\|_{V}^{r^{2} / r^{\prime}}+\|\mathbf{R}\|_{V}^{r}+\|R\|_{P}^{r^{\prime}}\right) .
\end{aligned}
$$


and

$$
\begin{aligned}
\|\Upsilon\|_{T}^{r^{\prime}} & \leq C\left(\left\|\sigma-\sigma^{N}\right\|_{T}^{r}+\left\|\mathbf{u}-\mathbf{u}^{N}\right\|_{V}^{r}\right) \\
\|\mathbf{R}\|_{V}^{r} & \leq C\left(\left\|\sigma-\sigma^{N}\right\|_{T}^{r^{\prime}}+\left\|p-p^{N}\right\|_{P}^{r^{\prime}}\right) \\
\|R\|_{P}^{r^{\prime}} & \leq C\left(\left\|\mathbf{u}-\mathbf{u}^{N}\right\|_{V}^{r}\right) .
\end{aligned}
$$

Proof: The proof of (3.11)-(3.13) uses the inf-sup conditions and properties A2 and A3.

Using (2.15),

$$
\begin{aligned}
\left\|\mathbf{u}-\mathbf{u}^{N}\right\|_{V} & \leq \frac{1}{\gamma_{V S}} \sup _{\tau \in T} \frac{b\left(\tau, \mathbf{u}-\mathbf{u}^{N}\right)}{\|\tau\|_{T}} \\
& =\frac{1}{\gamma_{V S}} \sup _{\tau \in T} \frac{a(\sigma, \tau)-a\left(\sigma^{N}, \tau\right)-\mathbb{A}(\Upsilon, \tau)}{\|\tau\|_{T}} \\
& \leq \frac{1}{\gamma_{V S}} \sup _{\tau \in T} \frac{a(\sigma, \tau)-a\left(\sigma^{N}, \tau\right)}{\|\tau\|_{T}}+\frac{1}{\gamma_{V S}} \sup _{\tau \in T} \frac{\mathbb{A}(\Upsilon, \tau)}{\|\tau\|_{T}}
\end{aligned}
$$

Applying Hölder's inequality we obtain

$$
\begin{aligned}
\sup _{\tau \in T} \frac{\mathbb{A}(\Upsilon, \tau)}{\|\tau\|_{T}} & =\sup _{\tau \in T} \frac{\int_{\Omega}|\Upsilon|^{r^{\prime}-2} \Upsilon: \tau d \Omega}{\|\tau\|_{T}} \\
& \leq \sup _{\tau \in T} \frac{\left(\int_{\Omega}\left(|\Upsilon|^{r^{\prime}-2}|\Upsilon|\right)^{r} d \Omega\right)^{1 / r}\left(\int_{\Omega}|\tau|^{r^{\prime}} d \Omega\right)^{1 / r^{\prime}}}{\|\tau\|_{T}} \\
& =\|\Upsilon\|_{T}^{r^{\prime} / r} .
\end{aligned}
$$

Combining (3.17) and (3.18) we have the following estimate

$$
\left\|\mathbf{u}-\mathbf{u}^{N}\right\|_{V} \leq \frac{1}{\gamma_{V S}} \sup _{\tau \in T} \frac{a(\sigma, \tau)-a\left(\sigma^{N}, \tau\right)}{\|\tau\|_{T}}+\frac{1}{\gamma_{V S}}\|\Upsilon\|_{T}^{r^{\prime} / r}
$$

From the velocity-pressure inf-sup condition

$$
\begin{aligned}
\left\|p-p^{N}\right\|_{P} & \leq \frac{1}{\gamma_{V P}} \sup _{\mathbf{v} \in V} \frac{s\left(\mathbf{v}, p-p^{N}\right)}{\|\mathbf{v}\|_{V}} \\
& =\frac{1}{\gamma_{V P}} \sup _{\mathbf{v} \in V} \frac{b\left(\sigma-\sigma^{N}, \mathbf{v}\right)-\mathbb{B}(\mathbf{R}, \mathbf{v})}{\|\mathbf{v}\|_{V}} \\
& \leq \frac{1}{\gamma_{V P}} \sup _{\mathbf{v} \in V} \frac{\left\|\sigma-\sigma^{N}\right\|_{T}\|\mathbf{v}\|_{V}}{\|\mathbf{v}\|_{V}}+\frac{1}{\gamma_{V P}} \sup _{\mathbf{v} \in V} \frac{\mathbb{B}(\mathbf{R}, \mathbf{v})}{\|\mathbf{v}\|_{V}}
\end{aligned}
$$

As above, using Hölder's inequality

$$
\begin{aligned}
\sup _{\mathbf{v} \in V} \frac{\mathbb{B}(\mathbf{R}, \mathbf{v})}{\|\mathbf{v}\|_{V}} & =\sup _{\mathbf{v} \in V} \frac{\int_{\Omega}|\nabla \mathbf{R}|^{r-2} \nabla \mathbf{R}: \nabla \mathbf{v} d \Omega}{\|\mathbf{v}\|_{V}} \\
& \leq \sup _{\mathbf{v} \in V} \frac{\left(\int_{\Omega}\left(|\nabla \mathbf{R}|^{r-2}|\nabla \mathbf{R}|\right)^{r^{\prime}} d \Omega\right)^{1 / r^{\prime}}\left(\int_{\Omega}|\nabla \mathbf{v}|^{r} d \Omega\right)^{1 / r}}{\|\mathbf{v}\|_{V}} \\
& =\|\mathbf{R}\|_{V}^{r / r^{\prime}} .
\end{aligned}
$$


Combining (3.20) and (3.21)

$$
\left\|p-p^{N}\right\|_{P} \leq \frac{1}{\gamma_{V P}}\left\|\sigma-\sigma^{N}\right\|_{T}+\frac{1}{\gamma_{V P}}\|\mathbf{R}\|_{V}^{r / r^{\prime}} .
$$

Next we bound $\left\|\sigma-\sigma^{N}\right\|_{T}$. Substituting $\tau=\sigma-\sigma^{N}$ into (3.5) and using (3.6) and (3.7)

$$
\begin{aligned}
a\left(\sigma, \sigma-\sigma^{N}\right)-a\left(\sigma^{N}, \sigma-\sigma^{N}\right)= & \mathbb{A}\left(\Upsilon, \sigma-\sigma^{N}\right)+b\left(\sigma-\sigma^{N}, \mathbf{u}-\mathbf{u}^{N}\right) \\
= & \mathbb{A}\left(\Upsilon, \sigma-\sigma^{N}\right)+\mathbb{B}\left(\mathbf{R}, \mathbf{u}-\mathbf{u}^{N}\right)+s\left(\mathbf{u}-\mathbf{u}^{N}, p-p^{N}\right) \\
= & \mathbb{A}\left(\Upsilon, \sigma-\sigma^{N}\right)+\mathbb{B}\left(\mathbf{R}, \mathbf{u}-\mathbf{u}^{N}\right)+\mathbb{S}\left(R, p-p^{N}\right) \\
\leq & \|\Upsilon\|_{T}^{r^{\prime} / r}\left\|\sigma-\sigma^{N}\right\|_{T}+\|\mathbf{R}\|_{T}^{r / r^{\prime}}\left\|\mathbf{u}-\mathbf{u}^{N}\right\|_{V}+\|R\|_{P}^{r^{\prime} / r}\left\|p-p^{N}\right\|_{P} \\
\leq & \|\Upsilon\|_{T}^{r^{\prime} / r}\left\|\sigma-\sigma^{N}\right\|_{T} \\
& +\|\mathbf{R}\|_{T}^{r / r^{\prime}}\left(\frac{1}{\gamma_{V S}} \sup _{\tau \in T} \frac{a(\sigma, \tau)-a\left(\sigma^{N}, \tau\right)}{\|\tau\|_{T}}+\frac{1}{\gamma_{V S}}\|\Upsilon\|_{T}^{r^{\prime} / r}\right) \\
& +\|R\|_{P}^{r^{\prime} / r}\left(\frac{1}{\gamma_{V P}}\left\|\sigma-\sigma^{N}\right\|_{T}+\frac{1}{\gamma_{V P}}\|\mathbf{R}\|_{V}^{r / r^{\prime}}\right) .
\end{aligned}
$$

Using the coercivity assumption (2.9),

$$
\begin{aligned}
a\left(\sigma, \sigma-\sigma^{N}\right)-a\left(\sigma^{N}, \sigma-\sigma^{N}\right) & =\int_{\Omega}\left(\breve{g}(\sigma) \sigma-\breve{g}\left(\sigma^{N}\right) \sigma^{N}\right):\left(\sigma-\sigma^{N}\right) d \Omega \\
& \geq c \int_{\Omega}\left|\sigma-\sigma^{N}\right|^{r^{\prime}} d \Omega \\
& =c\left\|\sigma-\sigma^{N}\right\|_{T}^{r^{\prime}} .
\end{aligned}
$$

Also,

$$
\begin{aligned}
a(\sigma, \tau)-a\left(\sigma^{N}, \tau\right) & =\int_{\Omega}\left(\breve{g}(\sigma) \sigma-\breve{g}\left(\sigma^{N}\right) \sigma^{N}\right): \tau d \Omega \\
& \leq \int_{\Omega}\left|\breve{g}(\sigma) \sigma-\breve{g}\left(\sigma^{N}\right) \sigma^{N}\right||\tau| d \Omega \\
& \leq\left(\int_{\Omega}\left|\breve{g}(\sigma) \sigma-\breve{g}\left(\sigma^{N}\right) \sigma^{N}\right|^{r} d \Omega\right)^{1 / r}\|\tau\|_{T}
\end{aligned}
$$

Using the continuity assumption (2.10),

$$
\begin{aligned}
\left(\int_{\Omega}\left|\breve{g}(\sigma) \sigma-\breve{g}\left(\sigma^{N}\right) \sigma^{N}\right|^{r} d \Omega\right)^{1 / r} & \leq\left(\int_{\Omega} M^{r}\left(|\sigma|+\left|\sigma^{N}\right|\right)^{\left(r^{\prime}-2\right) r}\left|\sigma-\sigma^{N}\right|^{r} d \Omega\right)^{1 / r} \\
& \leq M\left\||\sigma|+\left|\sigma^{N}\right|\right\|_{T}^{\left(r^{\prime}-2\right)}\left\|\sigma-\sigma^{N}\right\|_{T} \\
& \leq M\left(\|\sigma\|_{T}^{\left(r^{\prime}-2\right)}+\left\|\sigma^{N}\right\|_{T}^{\left(r^{\prime}-2\right)}\right)\left\|\sigma-\sigma^{N}\right\|_{T} .
\end{aligned}
$$

Thus,

$$
\sup _{\tau \in T} \frac{a(\sigma, \tau)-a\left(\sigma^{N}, \tau\right)}{\|\tau\|_{T}} \leq C\left(\|\sigma\|_{T}^{\left(r^{\prime}-2\right)}+\left\|\sigma^{N}\right\|_{T}^{\left(r^{\prime}-2\right)}\right)\left\|\sigma-\sigma^{N}\right\|_{T}
$$


Combining (3.23),(3.24),(3.27), together with the boundedness of $\sigma$ and $\sigma^{N}$, we have

$$
\begin{aligned}
c\left\|\sigma-\sigma^{N}\right\|_{T}^{r^{\prime}} \leq & C\left(\|\Upsilon\|_{T}^{r^{\prime} / r}\left\|\sigma-\sigma^{N}\right\|_{T}\right. \\
& +\|\mathbf{R}\|_{V}^{r / r^{\prime}}\left\|\sigma-\sigma^{N}\right\|_{T}+\|\Upsilon\|_{T}^{r^{\prime} / r}\|\mathbf{R}\|_{V}^{r / r^{\prime}} \\
& \left.+\|R\|_{P}^{r^{\prime} / r}\left\|\sigma-\sigma^{N}\right\|_{T}+\|\mathbf{R}\|_{V}^{r / r^{\prime}}\|R\|_{P}^{r^{\prime} / r}\right) .
\end{aligned}
$$

Applying Young's inequality then yields

$$
\left\|\sigma-\sigma^{N}\right\|_{T}^{r^{\prime}} \leq C\left(\|\Upsilon\|_{T}^{r^{\prime}}+\|\mathbf{R}\|_{V}^{r^{2} / r^{\prime}}+\|\mathbf{R}\|_{V}^{r}+\|R\|_{P}^{r^{\prime}}\right)
$$

Now, using (3.27),(3.28) in (3.19) and (3.22) we obtain

$$
\begin{aligned}
\left\|\mathbf{u}-\mathbf{u}^{N}\right\|_{V}^{r} & \leq C\left(\|\Upsilon\|_{T}^{r^{\prime}}+\|\Upsilon\|_{T}^{r}+\|\mathbf{R}\|_{V}^{r^{3} / r^{\prime 2}}+\|\mathbf{R}\|_{V}^{r^{2} / r^{\prime}}+\|R\|_{P}^{r}\right) \\
\left\|p-p^{N}\right\|_{P}^{r^{\prime}} & \leq C\left(\|\Upsilon\|_{T}^{r^{\prime}}+\|\mathbf{R}\|_{V}^{r^{2} / r^{\prime}}+\|\mathbf{R}\|_{V}^{r}+\|R\|_{P}^{r^{\prime}}\right)
\end{aligned}
$$

To establish estimates (3.14)-(3.16) we use (3.5)-(3.7).

$$
\begin{aligned}
& \mathbb{A}(\Upsilon, \Upsilon)=\|\Upsilon\|_{T}^{r^{\prime}}=a(\sigma, \Upsilon)-a\left(\sigma^{N}, \Upsilon\right)-b\left(\Upsilon, \mathbf{u}-\mathbf{u}^{N}\right) \\
& \leq M\left(\|\sigma\|_{T}^{\left(r^{\prime}-2\right)}+\left\|\sigma^{N}\right\|_{T}^{\left(r^{\prime}-2\right)}\right)\left\|\sigma-\sigma^{N}\right\|_{T}\|\Upsilon\|_{T}+\|\Upsilon\|_{T}\left\|\mathbf{u}-\mathbf{u}^{N}\right\|_{V} \\
& \Rightarrow\|\Upsilon\|_{T}^{r^{\prime}} \leq C\left(\left\|\sigma-\sigma^{N}\right\|_{T}^{r}+\left\|\mathbf{u}-\mathbf{u}^{N}\right\|_{V}^{r}\right) . \\
& \mathbb{B}(\mathbf{R}, \mathbf{R})=\|\mathbf{R}\|_{V}^{r}=b\left(\sigma-\sigma^{N}, \mathbf{R}\right)-s\left(\mathbf{R}, p-p^{N}\right) \\
& \leq\left\|\sigma-\sigma^{N}\right\|_{T}\|\mathbf{R}\|_{V}+\|\mathbf{R}\|_{V}\left\|p-p^{N}\right\|_{P} \\
& \Rightarrow\|\mathbf{R}\|_{V}^{r} \leq C\left(\left\|\sigma-\sigma^{N}\right\|_{T}^{r^{\prime}}+\left\|p-p^{N}\right\|_{P}^{r^{\prime}}\right) . \\
& \mathbb{S}(R, R)=\|R\|_{P}^{r^{\prime}}=s\left(\mathbf{u}-\mathbf{u}^{N}, R\right) \\
& \leq\left\|\mathbf{u}-\mathbf{u}^{N}\right\|_{V}\|R\|_{P} \\
& \Rightarrow\|R\|_{P}^{r^{\prime}} \leq C\left\|\mathbf{u}-\mathbf{u}^{N}\right\|_{V}^{r} \text {. }
\end{aligned}
$$

\section{Computation of A Posteriori Error Estimators}

In this section we discuss the computation of the a posteriori error estimators. The actual residual functions $\Upsilon, \mathbf{R}, R$, defined by the non-linear projection (3.5)-(3.7), do not need to be explicitly computed. The a posteriori error bounds (3.11)-(3.13) are given in terms of the norms of the residual functions, not the residual functions themselves. As shown below the computations of these norms turn out to be very natural, involving the residuals of the variational equations.

$\underline{\text { Evaluation of } \Upsilon_{T}}$ 
From the definition of $\Upsilon$, (3.5), and using (2.11), we have

$$
\begin{array}{rlrl}
\int_{\Omega}|\Upsilon|^{r^{\prime}-2} \Upsilon: \tau d \Omega & =a(\sigma, \tau)-b(\tau, \mathbf{u})-\left(a\left(\sigma^{N}, \tau\right)-b\left(\tau, \mathbf{u}^{N}\right)\right), \quad \forall \tau \in T \\
& =-\int_{\Omega}\left(\breve{g}\left(\sigma^{N}\right) \sigma^{N}-d\left(\mathbf{u}^{N}\right)\right): \tau d \Omega, \quad \forall \tau \in T & \\
\Rightarrow 0 & =\int_{\Omega}\left(|\Upsilon|^{r^{\prime}-2} \Upsilon+\breve{g}\left(\sigma^{N}\right) \sigma^{N}-d\left(\mathbf{u}^{N}\right)\right): \tau d \Omega, \quad \forall \tau \in T \\
\Rightarrow|\Upsilon|^{r^{\prime}-2} \Upsilon & =-\left(\breve{g}\left(\sigma^{N}\right) \sigma^{N}-d\left(\mathbf{u}^{N}\right)\right) .
\end{array}
$$

Taking the inner-product of both sides of (4.1) with themselves yields

$$
|\Upsilon|^{2 r^{\prime}-2}=\left(\breve{g}\left(\sigma^{N}\right) \sigma^{N}-d\left(\mathbf{u}^{N}\right)\right):\left(\breve{g}\left(\sigma^{N}\right) \sigma^{N}-d\left(\mathbf{u}^{N}\right)\right) .
$$

Hence, taking the $r / 2$ power of both sides of (4.2) and integrating over $\Omega$ we obtain

$$
\begin{aligned}
\|\Upsilon\|_{T}^{r^{\prime}} & =\int_{\Omega}|\Upsilon|^{r^{\prime}-2} \Upsilon: \Upsilon d \Omega \\
& =\int_{\Omega}\left[\left(\breve{g}\left(\sigma^{N}\right) \sigma^{N}-d\left(\mathbf{u}^{N}\right)\right):\left(\breve{g}\left(\sigma^{N}\right) \sigma^{N}-d\left(\mathbf{u}^{N}\right)\right)\right]^{r / 2} d \Omega,
\end{aligned}
$$

which represents $r^{\text {th }}$ power of the $L^{r}$ norm of residue of (2.11).

Evaluation of $R$

Proceeding analogously as for $\Upsilon$ we obtain

$$
\|R\|_{P}^{r^{\prime}}=\int_{\Omega}\left|\nabla \cdot \mathbf{u}^{N}\right|^{r} d \Omega
$$

which represents $r^{t h}$ power of the $L^{r}$ norm of residue of (2.13).

\section{Evaluation of $\mathbf{R}$}

From the definition of $\mathbf{R},(3.6)$, and using (2.12) and that $\sigma^{N}$ is a symmetric tensor, we have

$$
\begin{aligned}
\int_{\Omega}|\nabla \mathbf{R}|^{r-2} \nabla \mathbf{R}: \nabla \mathbf{v} d \Omega & =b(\sigma, \mathbf{v})-s(\mathbf{v}, p)-\left(b\left(\sigma^{N}, \mathbf{v}\right)-s\left(\mathbf{v}, p^{N}\right)\right), \quad \forall \mathbf{v} \in V \\
& =\int_{\Omega} f_{p} \nabla \cdot \mathbf{v} d \Omega-\int_{\Omega} \sigma^{N}: \nabla \mathbf{v} d \Omega+\int_{\Omega} \nabla \cdot \mathbf{v} p^{N} d \Omega, \quad \forall \mathbf{v} \in V \\
& =\int_{\Omega}\left(f_{p} I-\sigma^{N}+p^{N} I\right): \nabla \mathbf{v} d \Omega, \quad \forall \mathbf{v} \in V,
\end{aligned}
$$

which implies

$$
|\nabla \mathbf{R}|^{r-2} \nabla \mathbf{R}=f_{p} I-\sigma^{N}+p^{N} I .
$$

In (4.5) and (4.6) $I$ denotes the identity tensor.

Proceeding as above we obtain

$$
\|\mathbf{R}\|_{V}^{r}=\int_{\Omega}\left[\left(f_{p} I-\sigma^{N}+p^{N} I\right):\left(f_{p} I-\sigma^{N}+p^{N} I\right)\right]^{r^{\prime} / 2} d \Omega,
$$


which we recognize as the $r^{\prime t h}$ power of the $L^{r^{\prime}}$ norm of residue of (2.12).

In case $\mathbf{f}$ does not represent a conservative vector field we introduce the unique $\tilde{\mathbf{f}} \in\left(W_{0}^{1, r^{\prime}}(\Omega)\right)^{d}$ satisfying

$$
\int_{\Omega} \nabla \tilde{\mathbf{f}}: \nabla \mathbf{v} d \Omega=\int_{\Omega} \mathbf{f} \cdot \mathbf{v} d \Omega, \quad \forall \mathbf{v} \in V .
$$

(Note that for the approximation of $\tilde{\mathbf{f}}$, using the same test and trial spaces, (4.8) represents the inversion of a symmetric, positive definite operator. Hence, very efficient numerical schemes such as preconditioned conjugate gradient, and multigrid, may be used to accurately approximate $\tilde{\mathbf{f}}$ in a preliminary calculation.)

In place of (2.12) we use

$$
b(\sigma, \mathbf{v})-s(\mathbf{v}, p)=\int_{\Omega} f \cdot \mathbf{v} d \Omega, \quad \forall \mathbf{v} \in V
$$

$\|\mathbf{R}\|_{V}^{r}$ is then given by

$$
\|\mathbf{R}\|_{V}^{r}=\int_{\Omega}\left[\left(\nabla \tilde{\mathbf{f}}-\sigma^{N}+p^{N} I\right):\left(\nabla \tilde{\mathbf{f}}-\sigma^{N}+p^{N} I\right)\right]^{r^{\prime} / 2} d \Omega .
$$

\section{Conclusion}

A general a posteriori error estimator for a three-field model of a generalized Stokes problem has been proposed and analyzed. The estimator may be applied to a wide range of approximations, as only minimal assumptions are made regarding the approximation. The constants appearing in the upper and lower bound estimates are clearly identified. The components of the a posteriori error estimator are easily computable, and when appropriately combined, provide upper and lower bounds to the approximation error. However, the computed components do not directly relate to physical quantities. For example, $\|\Upsilon\|_{T}^{r^{\prime}}$ small does not imply that the physical quantity $\left\|\sigma-\sigma^{N}\right\|_{T}^{r^{\prime}}$ is necessarily small. Some new types of error estimators using quantities of physical interest have recently been developed, see $[3,18]$ and the references therein.

Acknowledgement The authors would like to acknowledge helpful comments made by Professor Hyesuk Lee, Department of Mathematical Sciences, Clemson University, and several useful suggestions made by the referee. 


\section{References}

[1] M. Ainsworth and J.T. Oden, A posteriori error estimators for the Stokes and Oseen equations, SIAM J. Numer. Anal., 34, 228-245, 1997.

[2] J. Baranger, K. Najib and D. Sandri, Numerical analysis of a three-fields model for a quasiNewtonian flow, Comput. Methods Appl. Mech. Engrg., 109, 281-292, 1993.

[3] R. Becker and R. Rannacher, An optimal control approach to a posteriori error estimation in finite element methods, Acta Numer., 10, 1-102, 2001.

[4] R.B. Bird, R.C. Armstrong, and O. Hassager, Dynamics of Polymeric Liquids, John Wiley and Sons, New York, 1987.

[5] C. Chauvière and R.G. Owens, How accurate is your solution? Error indicators for viscoelastic flow calculations, J. Non-Newtonian Fluid Mech., 95, 1-33, 2000.

[6] P.G. Ciarlet, The Finite Element Method for Elliptic Problems, North-Holland, Amsterdam, 1978 .

[7] G.N. Gatica, M. Gonzalez, and S. Meddahi, A low-order mixed finite element method for a class of quasi-Newtonian Stokes flows, Preprint 02-01, Department of Mathematics, University of Concepcion, (available at http://www.ing-mat.udec.cl/investigacion/publicaciones/paginaspp/pp02-01.html), 2002.

[8] M.I. Gerritsma and T.N. Phillips, Compatible spectral approximations for the velocity-pressurestress formulation of the Stokes problem, SIAM J. Sci. Comput., 20(4), 1530-1550, 1999.

[9] V. Girault and P.A. Raviart, Finite element methods for Navier-Stokes equations, SpringerVerlag, Berlin, Heidelberg, 1986.

[10] M.D. Gunzburger, Finite Element Methods for Viscous Incompressible Flows, Academic Press, Boston, 1989.

[11] C.D. Han, Multiphase Flow in Polymer Processing, Academic Press, New York, 1981.

[12] H. Jin, A subdomain residual method for a posteriori error estimates of finite element solutions of viscoelastic fluid flows, Comput. Methods Appl. Mech. Engrg., 130, 93-113, 1996.

[13] M. Maday, C. Mavriplis and A.T. Patera, Nonconforming mortar element methods: Application to spectral discretizations. In: Domain Decomposition Methods. Proceedings of the Second International Symposium on Domain Decomposition Methods, Los Angeles, California, January 14-16, 1988 (T.F. Chan, R. Glowinski, J. Periaux and O.B. Widlund, Eds.), SIAM, Philadelphia, 1989, 392-418.

[14] O.A. Ladyzhenskaya, New equations for the description of the viscous incompressible fluids and solvability in the large of the boundary value problems for them, Boundary Value Problems of Mathematical Physics V, American Mathematical Society, Providence RI, (1970).

[15] H. Manouzi and M. Farhloul, Mixed finite element analysis of a non-linear three-fields Stokes model, IMA J. Numer. Anal., 21, 143-164, 2001. 
[16] S.H. Momeni-Masuleh and T.N. Phillips, A posteriori error estimation for the velocity-pressurestress formulation of the Stokes problem, preprint Department of Mathematics, University of Wales, Aberystwyth, 2004.

[17] I. Mutlu, P. Townsend and M.F. Webster, Adaptive solutions for viscoelastic flows, Comm. Numer. Methods Engrg., 12, 643-655, 1996.

[18] J.T. Oden and S. Prudhomme, Goal-oriented error estimation and adaptivity for the finite element method, Computers Math. Applic., 41(5-6), 735-756, 2001.

[19] R.G. Owens, A posteriori error estimates for spectral element solutions to viscoelastic flow problems, Comput. Methods Appl. Mech. Engrg., 164, 375-395, 1998.

[20] R.G. Owens and T.N. Phillips, Computational Rheology, Imperial College Press, London, 2002.

[21] M. Picasso and J. Rappaz, Existence, a priori and a posteriori error estimates for a nonlinear three field problem arising from Oldroyd-B viscoelastic flows, Math. Model. Numer. Anal., 35(5), 879-897, 2001.

[22] R.R. Rao and B.A. Finlayson, Adaptive refinement of a viscoelastic flow problem with the explicitly elliptic momentum equation, J. Non-Newt. Fluid Mech., 38, 223-246, 1991.

[23] D. Sandri, A posteriori estimators for mixed finite element approximations of a fluid obeying the power law, Comput. Methods Appl. Mech. Engrg., 166, 329-340, 1998.

[24] R. Verfürth, A review of a posteriori error estimation and adaptive mesh refinement techniques, Wiley, Teubner, 1996.

[25] V. Warichet and V. Legat, Adaptive hp-finite element viscoelastic flow calculations, Comput. Methods Appl. Mech. Engrg., 136, 93-110, 1996.

[26] F. Yurun and M.J. Crochet, High order finite element methods for steady viscoelastic flows, $J$. Non-Newtonian Fluid Mech., 57, 283-311, 1995.

[27] E. Zeidler, Nonlinear Functional Analysis and its Applications II/B, Springer-Verlag, New York, 1990. 